\title{
Contribuciones empíRICAS PARA LA VALIDEZ DE GRUPOS CONTRASTADOS DE LA BATERía de TAREAS de AutorRegulación Cognitiva (TAC)*
}

\section{EMPIRICAL CONTRIBUTIONS FOR CONTRASTING GROUPS VALIDITY OF THE TAREAS DE AUtORREgUlaCión COGNITIVA (TAC) BATTERY}

\author{
María M. Richard's** SANTIAgo VernuCci*** Elana Zamora****, \\ LOREnA CANet JURIC ${ }^{* * * * *}$, ISABEL INTROZZI ${ }^{* * * * *}$ Y JOAN GUARDIA I $^{* * * * * *}$
}

\begin{abstract}
*Esta investigación ha sido financiada por el Consejo Nacional de Investigaciones Científicas y Técnicas (CONICET). ${ }^{* *}$ Doctora en Psicología. Miembro de la Carrera del Investigador Científico del Consejo Nacional de Investigaciones Científicas y Técnicas (CONICET). E-Mail: mariamartarichards@gmail.com Instituto de Psicología Básica, Aplicada y Tecnología - Facultad de Psicología. Universidad Nacional de Mar del Plata (UNMdP). Funes 3250, Cuerpo V, Nivel III. Mar del Plata, Argentina.

***Licenciado en Psicología. Becario Doctoral del Consejo Nacional de Investigaciones Científicas y Técnicas (CONICET). ****Licenciada en Psicología. Becaria Doctoral del Consejo Nacional de Investigaciones Científicas y Técnicas (CONICET). ${ }^{* * * * *}$ Doctora en Psicología. Miembro de la Carrera del Investigador Científico del Consejo Nacional de Investigaciones Científicas y Técnicas (CONICET).

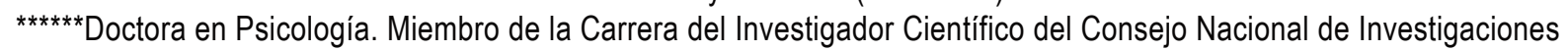
Científicas y Técnicas (CONICET).

*******Doctor en Psicología. Catedrático del Departamento de Metodología de las Ciencias del Comportamiento en la Facultad de Psicología de la Universidad de Barcelona (UB), España.

Los autores agradecen la colaboración para evaluar la muestra clínica a la Fundación ADANA, a su Presidente, la Dra. Isabel Rubió y a su Directora la Dra. Beatriz Mena como así también a la Asociación TDAH Valles y a su Presidente, la

Dra. Anna López. Asimismo, agradecen al Dr. Joan Guardia su tarea como supervisor extranjero y al estudiante avanzado Carlos Luces Iza, su tarea en la recolección de datos.
\end{abstract}

\section{RESUMEN}

Los déficit de las funciones ejecutivas (FE) suelen estar presentes en el Trastorno por Déficit de Atención e Hiperactividad (TDAH). Distintos trabajos mostraron algunas falencias vinculadas a la medición de las FE, como la participación de otros procesos que no constituyen objeto de la evaluación. En el trabajo que se informa se analizaron las diferencias existentes en las FE de niños con y sin diagnóstico de TDAH y se buscó identificar y establecer aquellas variables con mayor poder discriminante para la probabilidad de pertenencia a ambos grupos. Se utilizaron las tareas de la batería denominada Tareas de Autorregulación Cognitiva (TAC), diseñadas ad-hoc para evaluar específica y relativamente en forma independiente cada FE. Se trabajó con una muestra de 49 niños escolarizados de 7 a 12 años de edad, de ambos géneros, divididos en dos grupos, uno clínico de 19 niños con diagnóstico de TDAH, con una media de edad igual a 9.84 años $(D E=1.83)$ y uno control de 30 niños sin diagnóstico de TDAH con una media de edad igual a 10.27 años $(D E=.82)$, equiparados por género, edad, niveles socioeconómico y educacional. Los resultados mostraron que el análisis discriminante permitió clasificar correctamente al $98 \%$ de los participantes. Las ta- 
reas mejor discriminadas fueron la de Flexibilidad cognitiva y Control inhibitorio comportamental, con un $98.9 \%$ y $97.8 \%$, respectivamente, de casos correctos. Esto muestra que la TAC constituye una batería con adecuada sensibilidad y especificidad para discriminar y detectar casos de TDAH con un elevado nivel de confiabilidad y porcentaje de correctos clasificados.

Palabras clave: Funciones ejecutivas; Tareas de Autorregulación Cognitiva; TDAH; Validez de grupos contrastados; Curvas ROC.

\section{ABSTRACT}

The executive functions (EF) multidimensional approaches state that EFs consist of a set of processes with relative autonomy and independence: working memory (WM), cognitive flexibility (CF), and inhibition. Regarding the inhibitory operation, three dimensions are distinguished, each with distinct operative features: perceptual, cognitive, and behavioral inhibition. Perceptual inhibition is the process that allows the focus on relevant environmental stimuli through the attenuation of the interference generated by other stimuli present in the context. Cognitive inhibition is involved in the decreased level of activation of the prepotent mental representations of intrusive and irrelevant to the achievement of current goals. Finally, behavioral inhibition handles suppress or cancel behaviors and strong, prepotent and unsuitable behaviors. While this last type contributes to the inhibitory control of behavior, the other two processes (cognitive and perceptual inhibition) apply to cognition, as involved in regulating the perceptions and representations. Regarding executive operation in general, empirical evidence suggests that these dimensions are involved in such diverse fields as psychopathology, personality, emotion, attentional control and cognitive development. It has been found that deficits in EFs are usually present in the Attention Deficit Hyperactivity Disorder (ADHD). The common practice of treating children with ADHD as an undifferentiated group of participants in behavioral and neuropsychological research may have adverse methodological consequences. Relying on group averaging in comparing the performance of ADHD and control groups may produce misleading results, as it conceals possible effects that may characterize some but not all ADHD participants. According to the previous assumptions, the objectives of this work were (1) analyzing the EF performance in children with or without diagnosed ADHD and (2) identifying and setting the variables with greater discriminant power between the normal and clinical groups.

Battery tasks called Tareas de Autorregulación Cognitiva (TAC) ad-hoc and designed to assess EFs operation specifically and with relative independence with each other were used. The sample consisted of 49 school-attending children, aged 7 12 years, of both sexes, divided into two groups: (1) a clinical group of 19 children diagnosed with ADHD combined subtype ( $M$ age $=9.84$ years, $S D=1.83$ ), and (2) a control group of 30 children without ADHD diagnosis $(M$ age $=10.27$ years, $S D=.82$ ), matched by gender, age, socioeconomic and educational level. By analyzing the results, significant differences were found between verbal and visuospatial WM, CF, behavioral and perceptual inhibition indices. Then, a discriminant analysis was made in order to use the index values with significant differences and make predictions about the probability of a subject to be a member of a certain criteria variable value (clinical or control group condition).

Results show that discriminant analysis allows to correctly classifying $98 \%$ of the participants. The results indicated that the average RT of the mixed block (Cognitive Flexibility) and the Stop Signal RT (Behavioral Inhibition) are the indices with better sensitivity and specificity for detecting the presence of ADHD in this sample. Considering that the area under the curve indicates the probability to classify correctly a couple of healthy and ill individuals randomly selected, then it is possible to conclude that the values of Cognitive Flexibility and Behavioral Inhibitory control tasks had better discriminant power, as they correctly classified $98.9 \%$ and $97.8 \%$ of the total cases, respectively. This result shows that the TAC Battery presents adequate sensitivity and specificity to discriminate and detect ADHD with a high level of reliability and classification accuracy.

Summarizing, we highlight the importance of having an assessment battery such as the totally computerized TAC, which allows the assessment of EFs independently, with an attractive design and 
straightforward administering and scoring procedures.

Key words: Executive functions; ADHD; Contrasting groups validity; ROC curves.

\section{INTRODUCCIÓN}

Las funciones ejecutivas (FE) conforman un conjunto de procesos cognitivos de alto orden, involucrados en el control deliberado y voluntario del comportamiento, el pensamiento y las emociones, orientado al cumplimiento de objetivos (Diamond, 2013). La memoria de trabajo (MT), el control inhibitorio (CI) y la flexibilidad cognitiva (FC) se han identificado como las principales FE (Miyake \& Friedman, 2012).

La MT constituye un proceso clave para la cognición de alto nivel, dado que juega un papel fundamental en la comprensión lectora (Cain, Oakhill \& Bryant, 2004), la inteligencia fluida (Conway, Cowan, Bunting, Therriault \& Minkoff, 2002) y las habilidades matemáticas (Bull \& Lee, 2014). La FC facilita la autorregulación en diferentes dominios, al posibilitar la alternancia entre comportamientos conducentes a objetivos contrapuestos (Hofmann, Schmeichel \& Baddeley, 2012). El CI se ha vinculado al logro de diversas capacidades como la atención, el desempeño académico y la regulación emocional (Mischel et al., 2011). Las FE han demostrado además estar implicadas no sólo en el funcionamiento y desarrollo normal, sino también en distintos trastornos psicopatológicos (ver Diamond, 2013).

Uno de los trastornos con mayor compromiso de las FE y de mayor prevalencia en población infantil es el Trastorno de Déficit de Atención e Hiperactividad (TDAH - Barkley, 1997; Scahill \& Schwab-Stone, 2000) y está caracterizado por el déficit atencional (distractibilidad) y la falta de control sobre los impulsos (hiperactividad e impulsividad) (American Psychiatric Association, 2013). El estudio de las FE en niños con TDAH se ha centrado en la inhibición comportamental (Brocki \& Bohlin, 2006; Nigg, 2001) como la causa principal de los déficit observados en este trastorno (Barkley, 1997; Fuggetta, 2006). Estudios recientes que utilizaron tareas Stop Signal, la establecen como el principal factor que distingue a niños con y sin TDAH (Mc Auley, Crosbie, Charach \& Schachar, 2014; Senderecka, Grabowska, Gerc, Szewczyk \& Chmylak, 2012).

\section{LA INHIBICIÓN COMO CONSTRUCTO MULTIDIMENSIO- NAL}

En los últimos años han surgido modelos que proponen la existencia de varios procesos inhibitorios además de la inhibición comportamental (Friedman \& Miyake, 2004; Gandolfi, Viterbori, Traverso \& Usai, 2014; Howard, Johnson \& Pascual-Leone, 2014; Vadaga, Blair \& Li, 2016). Desde estos enfoques se plantea la necesidad de fragmentar el constructo en un conjunto de mecanismos con propiedades y características funcionales bien discriminadas. Brevemente, la inhibición perceptual sería el proceso responsable de suprimir estímulos irrelevantes del ambiente; la inhibición cognitiva tiene como función disminuir las representaciones de carácter intrusivo e irrelevantes para el logro de las metas actuales; la inhibición comportamental es la responsable de suprimir respuestas o impulsos prepotentes e inapropiados para la actividad en curso (Diamond, 2013). En consonancia con este enfoque, distintos autores han planteado la necesidad de analizar otros tipos inhibitorios además de la inhibición comportamental en el TDAH (Bédard, Trampush, Newcorn \& Halperin, 2010; Engelhardt, Nigg, Carr \& Ferreira, 2008).

En función de los desarrollos teóricos y empíricos sobre la estructura multidimensional de las FE y en particular del control inhibitorio, investigadores de la Universidad Nacional de Mar del Plata han diseñado una batería informatizada denominada Tareas de Autorregulación Cognitiva (TAC), integrada por tareas desarrolladas en base a paradigmas experimentales clásicos de la Psicología 
Cognitiva y Experimental. Su característica principal reside en que se ha intentado reducir al máximo la participación de otros procesos. Los estudios destinados a analizar las propiedades psicométricas de la TAC se realizaron con niños de desarrollo típico $(\mathrm{Ca}-$ net Juric, Introzzi \& Burin, 2015; Introzzi, Canet Juric, Montes, López \& Mascarello, 2015; Richard's, Introzzi, Zamora \& Vernucci, 2016), de allí la necesidad de analizar las propiedades psicométricas en población clínica con el trastorno de mayor prevalencia en población infantil.

De acuerdo con lo expuesto, los objetivos del estudio que se informa fueron analizar el desempeño en distintas FE en niños con y sin diagnóstico de TDAH e identificar y establecer las variables con mayor poder discriminante entre los grupos clínico y control.

\section{Metodología}

\section{PARTICIPANTES}

Se trabajó con una muestra no probabilística de 49 niños escolarizados de 7 a 12 años de edad (30.6\% de mujeres y $69.4 \%$ de varones) asignados a dos grupos: uno clínico (con diagnóstico de TDAH subtipo combinado) conformado por 19 niños, con una media de edad igual a 9.84 años y un desvío estándar igual a 1.83, pertenecientes a centros de atención especializados de la ciudad de Barcelona, el $21.1 \%$ eran mujeres y el $78.9 \%$, varones) de nivel socioeconómico medio (Índice de Status Social de Hollingshead - Navarro-Guzmán, 2005). Y un grupo control que era una muestra incidental de 30 alumnos de una institución educativa de nivel primario, sin patologías, de nivel socioeconómico medio, apareados por edad $(M=10.27$ años; $D E=.83)$, el $36.7 \%$ eran mujeres y el $63.3 \%$, varones.

Para garantizar la comparabilidad de los grupos se consideró el cumplimiento de una serie de condiciones. El grupo clínico se conformó según los siguientes criterios: (a) con diagnóstico de TDAH según DSM-V, (b) rango de edad entre 7 y 12 años, (c) con eva- luación neuropsicológica que confirme el diagnóstico realizado por el mismo equipo de profesionales, (d) nivel intelectual promedio y (e) encontrarse bajo escolaridad primaria normal.

Los criterios para la muestra control fueron: (a) no cumplir con el criterio diagnóstico para el TDAH según DSM-V, (b) rango de edad entre 7 y 12 años, (c) contar con evaluación neuropsicológica que descarte diagnóstico y (d) nivel intelectual promedio. Adicionalmente, se aplicó la prueba $t$ de diferencia de medias para muestras independientes, no se encontraron diferencias estadísticamente significativas entre ambos grupos en las variables de edad, género y nivel socioeconómico $(t=1.10, p>.05)$, lo que aseguró su comparabilidad.

\section{INSTRUMENTOS}

Inhibición perceptual: Es una tarea basada en el paradigma de Búsqueda Visual Conjunta (Treisman \& Gelade, 1980). Se le solicita al participante que señale la presencia o la ausencia de un estímulo target entre un conjunto variable de estímulos distractores. Todos los distractores comparten una característica con el target (forma o color), generando el efecto de interferencia necesario para la activación de la inhibición perceptual. La tarea está compuesta por un bloque de 10 ensayos de práctica, seguido de tres bloques experimentales de 40 ensayos cada uno. En cada bloque, los ensayos se distribuyen en cuatro condiciones según el número de distractores: 4, 8, 16 y 32. En cada ensayo, el participante debe responder lo más rápido y preciso posible presionando una tecla del teclado, según si el target está presente o ausente. Cuanto mayores sean los tiempos de respuesta (TR) y el porcentaje de errores, menor será la eficiencia del proceso.

Inhibición cognitiva: Esta tarea está basada en el paradigma experimental modificado de Sternberg (Oberauer, 2001). Los participantes deben completar un bloque de evaluación de 32 ensayos. Cada ensayo consiste 
en tres presentaciones separadas: aprendizaje, señal y reconocimiento. (1) Aprendizaje: se presentan simultáneamente dos filas de formas abstractas localizadas en la mitad superior e inferior de la pantalla. La longitud de la lista varía aleatoriamente entre uno y tres estímulos. (2) Señal: cuando desaparecen las listas, se presenta una señal que informa a los participantes cuál de las dos listas será relevante para una posterior tarea de reconocimiento. (3) Reconocimiento: al final aparece un estímulo blanco dentro de un rectángulo, los participantes deben indicar lo más rápido y con la mayor precisión posible, si la forma abstracta integraba o no la lista relevante.

Inhibición comportamental: Es una tarea basada en el Paradigma Stop Signal (Logan, 1994). Está integrada por dos bloques de práctica de 32 ensayos cada uno y un bloque experimental de 128 ensayos. En el primer bloque se presentan solo ensayos de ejecución (tarea primaria), seguido de un bloque de práctica, donde el participante debe realizar la misma tarea que en el bloque anterior, pero en este caso se solicita que intente detener su respuesta (presionar una tecla) cada vez que escuche una señal auditiva (señal de parar). Durante la ejecución de la tarea primaria, de manera imprevista el sujeto debe frenar su respuesta (ensayos stop-tarea secundaria) cada vez que escuche la señal de parar, que aparece en el $25 \%$ de los ensayos y a distintos intervalos luego de la presentación de la flecha (intervalos de la señal de parar-ISP-). El ISP se ajusta en función del desempeño del sujeto. Este procedimiento permite el cálculo del Tiempo de Frenado (TF), definido como la medida que refleja el tiempo de demora para detener la respuesta en los ensayos stop, principal índice de la tarea. El tercer bloque es el experimental, permite obtener los distintos índices de desempeño para el cálculo del TF. Se presentan de forma aleatoria $75 \%$ ensayos de ejecución y $25 \%$ de ensayos de parar.

Memoria de trabajo: Son tareas basadas en el paradigma dual (Hale, Bronik \& Fry, 1997). Las tareas duales requieren la ejecu- ción de una tarea primaria que implica el mantenimiento de un estímulo en la memoria a corto plazo y de una tarea secundaria que genera interferencia y tiene como objetivo interrumpir cualquier estrategia que pudiera facilitar el mantenimiento de la información de la tarea primaria. Las tareas utilizadas para la medición de este proceso fueron: la tarea de MT verbal con interferencia intradominio y la tarea de MT visoespacial con interferencia intradominio (ver Canet Juric et al., 2015). En la tarea de MT verbal se presenta secuencialmente una serie de dígitos de diferentes colores. En la tarea secundaria, los participantes deben decir en voz alta el color de cada número e inmediatamente desaparece de la pantalla y deben seleccionar con el cursor los dígitos presentados, en el orden correcto. La tarea de MT visoespacial consiste en la presentación de una matriz de $4 \times 4$ celdas, en la que aparecen secuencialmente estímulos (X) de diferentes colores. En la tarea secundaria, los participantes deben indicar el color de cada X señalando con el cursor una paleta de colores colocada a la derecha del estímulo presentado y señalar en una matriz vacía el orden en que apareció cada X. Ambas tareas se interrumpen cuando el participante indica dos secuencias incorrectas de manera consecutiva y se obtiene la estimación de amplitud de la tarea.

Flexibilidad cognitiva: Esta tarea es una versión modificada de la Tarea de las Flechas (ver Davidson, Amso, Anderson \& Diamond, 2006). Está compuesta por un bloque inicial de práctica de ocho ensayos, previo a cada uno de los bloques experimentales (congruente, incongruente y mixto), compuestos por 32 ensayos cada uno. En el bloque congruente, aparece en el lateral izquierdo o derecho de la pantalla, una mano con un dedo que señala recto hacia abajo que indica al participante que debe presionar la tecla ipsilateral al sitio en que se presenta el estímulo. En el bloque incongruente, aparece un dedo que señala diagonalmente hacia el lado opuesto, en el lateral izquierdo o derecho de la pantalla. En este caso, el participante debe presionar la tecla contralateral al lado en que se presenta el 
estímulo. En el bloque mixto se presentan 32 ensayos: 16 congruentes y 16 incongruentes. Los estímulos se distribuyen aleatoriamente respetando las siguientes condiciones: 16 estímulos en el lateral derecho (8 congruentes y 8 incongruentes) y 16 en el lateral izquierdo (8 congruentes y 8 incongruentes). La prueba requiere un cambio veloz entre dos tipos de reglas incompatibles (presionar del mismo lado o del lado opuesto al que aparece el estímulo). En este bloque se obtienen las siguientes medidas: porcentaje medio de respuestas correctas y TR medios.

\section{DISEÑO Y PROCEDIMIENTO}

En este estudio empírico se utilizó un diseño instrumental - transversal (Montero \& León, 2007).

Las evaluaciones fueron realizadas individualmente por profesionales especialmente entrenados de los Centros Especializados de TDAH: Fundación ADANA y Asociación TDAH Valles. El orden de administración estuvo contrabalanceado. Se solicitó el consentimiento informado de los padres y / o tutores y del niño para participar en el estudio. Para implementar esta investigación se tomaron en cuenta los lineamientos éticos que comprenden las actividades destinadas a obtener conocimientos sobre procesos psicológicos en seres humanos, recomendados por la American Psychological Association, los principios establecidos por la Convención Internacional sobre los Derechos del Niño y los lineamientos dados por CONICET para el comportamiento ético en las Ciencias Sociales y Humanidades.

\section{ANÁLISIS DE DATOS}

El plan de análisis se estructuró en tres etapas, en función de los objetivos. En primer lugar, se obtuvieron los principales índices de desempeño para cada tarea (ver Cuadro 1).

Luego se realizó un análisis discriminante para estimar cuáles eran las variables con mayor poder de clasificación con respecto al grupo clínico. Esta técnica permite determinar cuáles son las variables que más diferencian a los grupos, cuáles son relevantes a efectos de clasificar los sujetos.

Posteriormente y para analizar la validez clínica de las tareas, así como los indicadores de especificidad y sensibilidad para determinar la pertenencia al grupo clínico, se utilizó la Curva ROC (Receiver Operating Characteristic). Este tipo de representación simple y gráficamente permite interpretar la capacidad de discriminación de las tareas en todo el rango de puntos de corte y además los estimadores de sensibilidad y especificidad de las tareas (Hajian-Tilaki, 2013).

\section{Resultados}

Inicialmente se obtuvieron los principales índices de desempeño para cada tarea y se compararon los valores de ambos grupos (ver Tabla 1). Como puede verse, se encontraron diferencias significativas en los índices de las tareas de MT verbal y MT visoespacial, FC, inhibición comportamental e inhibición perceptual.

En segundo lugar, se realizó un análisis discriminante con el objeto de utilizar los valores observados de los índices con diferencias significativas (MT verbal, MT visoespacial, inhibición comportamental: TF, inhibición perceptual: TR medio 32 distractores, FC: TR medio en BM, precisión en BM, y coste de cambio local en TR) para realizar predicciones sobre la probabilidad de pertenencia de los sujetos a los valores de la variable criterio (condición de grupo clínico y control).

En la Tabla 2 y a partir de la función Lambda de Wilks se puede observar que las medias de las distintas medidas ejecutivas presentan diferencias significativas entre los grupos planteados. Como no se comprobó la igualdad de variancias $(M$ de Box $=97.965$; $p<.001)$ se procedió a realizar el análisis discriminante con estimación separada de la matriz de variancias-covariancias. La única función canónica discriminante indica que la función lineal discrimina bien, ya que pre- 
senta un autovalor de 5.33 y una correlación canónica de .918 (el 91.8\%) entre los grupos clínico y control de niños. El conjunto de las puntuaciones de las medidas ejecutivas de amplitud de MT verbal y MT visoespacial, tiempo de frenado de la tarea de inhibición comportamental, TR medios en la condición de 32 distractores de la tarea de inhibición perceptual, TR medio y Precisión en el bloque mixto, así como Coste de cambio local en TR en la tarea de FC, presentan diferencias significativas entre los grupos clínico y control $(\Lambda=.158, p<.001)$.

En la Tabla 3 se presentan los resultados de clasificación, los cuales muestran un 98\% de casos totales clasificados correctamente de los casos agrupados originales, con un $100 \%$ de verdaderos positivos para el grupo control y un $94.7 \%$ para el grupo clínico, lo que se considera como porcentajes destacados.

Posteriormente, se obtuvieron las curvas ROC generadas para representar la sensibilidad y la especificidad de la ecuación para todos los posibles puntos de corte. En este caso, la sensibilidad está representada por la capacidad para detectar TDAH cuando está presente, mientras que la especificidad se refiere a la capacidad para descartar el trastorno en los individuos sanos (Hajian-Tilaki, 2013). La capacidad de discriminación de la prueba diagnóstica puede evaluarse estimando el intervalo de confianza del área bajo la curva $(\mathrm{ABC})$. Si el intervalo no incluye el valor .5 , la prueba es capaz de discernir entre enfermos y sanos (Swets, 1988). El ABC equivale a la probabilidad de que la tarea identifique correctamente a dos niños pertenecientes a cada grupo, si uno de ellos fuera extraído aleatoriamente del grupo control y el otro, aleatoriamente del grupo clínico. Por esta razón, el ABC resulta una herramienta de gran utilidad para cuantificar la eficacia diagnóstica de las tareas (Zhou, Obuchowski \& McClish, 2002). Los resultados muestran que los índices con mayor poder discriminante, en función del $\mathrm{ABC}$ y los intervalos de confianza (ver Tabla 4) son los siguientes: el TR medio del bloque mixto de la tarea de $\mathrm{FC}(\mathrm{ABC}=.989, p<.001)$, se- guido del TF de la tarea de inhibición comportamental $(\mathrm{ABC}=.978, p<.001)$ y la precisión en el bloque mixto de la tarea de $\mathrm{FC}(\mathrm{ABC}=.907, p<.001)$.

En las Figuras 1, 2 y 3 se presentan las curvas ROC para los diferentes procesos.

Finalmente, en función de los coeficientes del ABC para cada medida analizada, se estimaron los puntos de corte óptimos para la probabilidad de pertenencia al grupo clínico, junto con los valores de sensibilidad y especificidad que cada tarea puede alcanzar simultáneamente. Para determinar los puntos de corte óptimos, el criterio utilizado fue la detección de los valores correspondientes al par que presenta simultáneamente la mayor sensibilidad y especificidad. Este valor corresponde al punto de la curva ROC que más se acerca al vértice superior izquierdo del gráfico. En la Tabla 5 se presentan los coeficientes del ABC para cada índice de desempeño, estimados con una técnica no paramétrica, junto con los puntos de corte y sus correspondientes valores de sensibilidad y especificidad.

En función de los resultados obtenidos, los puntos de corte del TR medio del bloque mixto de la tarea de FC, del TF de la tarea de inhibición comportamental y la precisión en el bloque mixto de la tarea de FC son los que presentan una mayor sensibilidad diagnóstica.

\section{Discusión}

El primer objetivo del estudio realizado fue analizar el desempeño en distintas FE en niños con y sin diagnóstico de TDAH. Para ello se evaluaron conjuntamente la MT, los procesos inhibitorios y la FC en una muestra de niños con desarrollo típico y con TDAH. Los resultados mostraron que existen diferencias significativas entre el grupo control y el grupo clínico, en el desempeño en las tareas que evalúan MT, inhibición comportamental, inhibición perceptual y FC.

Con respecto a la MT, este estudio se encuentra en consonancia con investigaciones que destacan el compromiso de este proceso 
en niños con TDAH (ver Doyle, 2006). En este sentido, tanto en la tarea de MT verbal como visoespacial se encontraron desempeños significativamente inferiores en el grupo de niños con TDAH en comparación con los controles. Constituye un aspecto relevante que las diferencias en el desempeño sean consistentes en ambas tareas, ya que puede suponerse una capacidad reducida de la MT en los niños con TDAH, y no un compromiso específico en el manejo de estímulos verbales o visoespaciales.

Los resultados en el desempeño de los niños con TDAH en las distintas tareas inhibitorias mostraron que en estos procesos tuvo un carácter diferencial. Tanto en las tareas que evalúan la inhibición comportamental como la inhibición perceptual, el rendimiento de los niños del grupo clínico fue significativamente menor que el de los niños del grupo control, mientras que en la inhibición cognitiva no se encontraron diferencias entre los grupos.

Los resultados obtenidos en la inhibición comportamental están en línea con el cuerpo de evidencia empírica que señala el compromiso de este proceso. En un meta-análisis que comparaba estudios realizados en niños con TDAH usando la Tarea Tipo Stop Signal (el mismo paradigma que se utilizó en el presente trabajo), se encontró que en un $82 \%$ de estos estudios se presentaba un déficit en el desempeño en esta tarea, poniendo de manifiesto el compromiso del control inhibitorio comportamental en este trastorno (Willcutt, Doyle, Nigg, Faraone \& Pennington, 2005).

Con respecto a la tarea de inhibición perceptual, los niños con TDAH mostraron una disminución en el rendimiento, en comparación con los niños del grupo control, lo que solo tuvo lugar en la condición que requiere una mayor demanda inhibitoria por la gran cantidad de distractores a inhibir (32).

Los resultados obtenidos en el desempeño en la tarea de inhibición cognitiva son consistentes con diferentes estudios que encuentran un funcionamiento normal en la supresión de representaciones mentales en esta clase de tareas en niños con TDAH (Engelhardt et al., 2008; Rubia, Smith, Taylor \&
Brammer, 2007). Teniendo en cuenta el modelo tripartito de la inhibición (perceptual, cognitiva y comportamental) sobre el que se desarrolla la Batería TAC, estos resultados son relevantes ya que muestran un compromiso diferencial según el tipo de proceso inhibitorio considerado.

En relación con la $\mathrm{FC}$, el grupo clínico presentó un nivel de desempeño inferior con respecto al grupo control. Esto se observó en mayores TR medios y en una precisión menor en la ejecución, así como en el coste de cambio expresado en los TR medios significativamente más lentos. En líneas generales, esta evidencia resulta consistente con los estudios que señalan las dificultades de alternar flexiblemente entre reglas en población con TDAH (Arán Filippetti \& Mías, 2009; Rubiales, 2014). Por ejemplo, Romero-Ayuso, Maestú, González-Marqués, Romo-Barrientos y Andrade (2006) estudiaron el desempeño ejecutivo según subtipos del trastorno, evidenciando un peor rendimiento en la tarea Simón y una mayor impulsividad en el grupo combinado de TDAH.

En síntesis, y en consonancia con estudios previos que señalan la existencia de un perfil heterogéneo de funcionamiento ejecutivo en niños con esta patología (Barkley, Edwards, Laneri, Fletcher \& Metevia, 2001; Roberts, Martel \& Nigg, 2013), en el trabajo que se informa se encontró que los niños con TDAH se caracterizan por exhibir un perfil de desempeño ejecutivo marcado por dificultades en el almacenamiento y procesamiento de estímulos verbales y espaciales, en la inhibición de respuestas prepotentes, en la inhibición de distractores perceptuales y en la alternancia entre reglas ante las demandas cambiantes de las tareas.

Con respecto al segundo objetivo de este trabajo, referido a la identificación de aquellas variables con mayor poder discriminante entre los grupos clínico y control, los resultados obtenidos muestran que las tareas de inhibición comportamental y FC son aquellas que presentan los índices de mayor peso para la detección de TDAH. Específicamente, se encontró que para poder clasificar a los niños con TDAH, los índices en cuyo desempeño se 
manifestaron diferencias entre los niños de ambos grupos, contribuyeron significativamente a la discriminación de los grupos, resultando en un $98 \%$ de casos clasificados correctamente. Este es un valioso aporte, dado que muestra con claridad que las tareas utilizadas en este trabajo, diseñadas para la evaluación independiente de cada FE, permiten clasificar con elevado grado de confianza el grupo de pertenencia. Además, todas las tareas (excepto la que evalúa inhibición cognitiva) contribuyen significativamente al proceso clasificatorio, lo que permitiría considerar que para evaluar el compromiso ejecutivo en el TDAH es necesario utilizar tareas que evalúen distintos procesos.

Se generaron y analizaron las curvas ROC correspondientes. Los resultados indicaron que el TR medio del bloque mixto (FC), la precisión en el bloque mixto (FC) y el TF (inhibición comportamental) son los índices con mejores valores de sensibilidad y especificidad de la TAC para detectar la presencia de TDAH en esta muestra. Si se considera que el $\mathrm{ABC}$ indica la probabilidad de clasificar correctamente un par de individuos sano y enfermo seleccionados aleatoriamente, entonces es posible concluir que los valores obtenidos por las pruebas de FC y de inhibición comportamental son destacados $(\mathrm{ABC}$, sensibilidad y especificidad mayor al $90 \%$ ), ya que tienen una alta probabilidad de ofrecer una clasificación correcta. Tomados en conjunto, los resultados obtenidos indican que la TAC constituye una batería con adecuada capacidad de discriminación y detección de casos clínicos de TDAH. Se considera que constituye un aporte valioso en el ámbito clínico dado que este es el trastorno con mayor prevalecía en población infantil, y además la identificación de los puntos de corte de cada proceso ejecutivo más discriminante posibilita a los psicólogos clínicos el diseño de estrategias e intervenciones específicas para cada grupo, basadas en el desem- peño de los niños en dos índices de Flexibilidad Cognitiva (TR medio y precisión del bloque mixto) y en el TF de la Tarea de Inhibición Comportamental.

Sin embargo, es necesario considerar algunas limitaciones del presente estudio, las cuales deberían abordarse para poder establecer con mayor seguridad la utilidad de las tareas ejecutivas de la Batería TAC en el diagnóstico de TDAH en población infantil. En primer lugar, sería necesario contar con una mayor cantidad de participantes, tanto en el grupo clínico como en el de niños sin el trastorno para una mejor generalización de los resultados. En segundo lugar, el rango de edad de los participantes (7 a 12 años) resulta amplio considerando el momento del ciclo vital en el que se encuentran, por lo que sería necesario reducir esta variabilidad. Una manera posible de abordar esta cuestión sería analizar el comportamiento de las tareas ejecutivas formando distintos grupos de edad, que no pudo llevarse a cabo debido al tamaño muestral disponible.

En función de estas limitaciones, próximos estudios que consideren las propiedades psicométricas de las tareas de la Batería TAC deberían intentar responder si las diferencias en el desempeño observadas en el presente trabajo son consistentes a lo largo del desarro1lo, o bien si se presentan diferencias en edades específicas, y en relación a esto último, si existen procesos que permiten diferenciar mejor a los niños con desarrollo típico de aquellos que poseen el trastorno en momentos determinados del ciclo vital.

Para finalizar, se destaca la importancia de contar con una batería de evaluación como la TAC completamente informatizada, que permita evaluar las FE de manera independiente, con un diseño atractivo y de fácil administración y puntuación y que además cuenta con valores de sensibilidad y especificidad para esta muestra clínica. 


\begin{tabular}{|c|c|}
\hline Funciones ejecutivas & Tarea e índices de medida \\
\hline $\begin{array}{l}\text { Memoria de trabajo: Capacidad para mantener infor- } \\
\text { mación de forma activa en la mente por un breve perí- } \\
\text { odo de tiempo con el objeto de completar una tarea, } \\
\text { registrar y almacenar información o generar objetivos. }\end{array}$ & $\begin{array}{l}\text { Tarea dual verbal (interferencia intradominio): Amplitud } \\
\text { de dígitos. } \\
\text { Tarea dual viso-espacial (interferencia intradominio): } \\
\text { Amplitud de localizaciones. }\end{array}$ \\
\hline $\begin{array}{l}\text { Inhibición perceptual: Proceso que permite focalizar la } \\
\text { atención en los estímulos relevantes y suprimir el } \\
\text { efecto de interferencia generado por los estímulos irre- } \\
\text { levantes del ambiente. }\end{array}$ & $\begin{array}{l}\text { Tarea de búsqueda visual conjunta: Diferencias entre } \\
\text { porcentaje medio de aciertos entre la condición de } 4 \text { y } \\
\text { de } 32 \text { distractores. Diferencias entre TRs medios de } \\
\text { aciertos entre la condición de } 4 \text { y de } 32 \text { distractores. } \\
\text { Precisión y TR medio en la condición de } 32 \text { distracto- } \\
\text { res. }\end{array}$ \\
\hline $\begin{array}{l}\text { Inhibición cognitiva: Proceso cuya principal función } \\
\text { consiste en controlar las representaciones mentales } \\
\text { prepotentes o intrusivas que son irrelevantes e inter- } \\
\text { fieren con la actividad en curso. }\end{array}$ & $\begin{array}{l}\text { Tarea de formas abstractas: Diferencias entre porcen- } \\
\text { taje medio de aciertos entre ítems nuevos e irrelevan- } \\
\text { tes / Diferencias entre TRs medios entre ítems nuevos } \\
\text { e irrelevantes. }\end{array}$ \\
\hline $\begin{array}{l}\text { Inhibición comportamental: Proceso encargado de } \\
\text { controlar el comportamiento a través de la supresión } \\
\text { de las respuestas prepotentes e impulsos que tienden } \\
\text { a imponerse con fuerza y que resultan inapropiados } \\
\text { para el logro de nuestros objetivos. }\end{array}$ & Tarea Stop Signal: Tiempo de frenado. \\
\hline $\begin{array}{l}\text { Flexibilidad cognitiva: Capacidad para cambiar rápida- } \\
\text { mente de una respuesta a otra mediante estrategias } \\
\text { alternativas, haciendo posible el cambio de perspecti- } \\
\text { vas de un problema y el ajuste a nuevas demandas, } \\
\text { reglas o prioridades. }\end{array}$ & $\begin{array}{l}\text { Tarea de los dedos: TR medio y precisión de las res- } \\
\text { puestas en el BM. Coste de cambio local en el BM, en } \\
\text { TR y Precisión. }\end{array}$ \\
\hline
\end{tabular}


TABLA 1

Prueba $t$ de Diferencia de Medias de los PROCESOS EJeCUTIVOS ENTRE LOS GRUPOS CLíniCO Y CONTROL

\begin{tabular}{|c|c|c|c|c|c|c|c|c|}
\hline Proceso & Índice & Grupo & $n$ & $M$ & $D E$ & $\begin{array}{l}\text { Error Est. } \\
\text { de Media }\end{array}$ & $t$ & $d$ \\
\hline MT verbal & Amplitud & $\begin{array}{l}\text { Control } \\
\text { Clínico }\end{array}$ & $\begin{array}{l}30 \\
19\end{array}$ & $\begin{array}{l}4.44 \\
3.47\end{array}$ & $\begin{array}{l}1.30 \\
.96\end{array}$ & $\begin{array}{l}.24 \\
.22\end{array}$ & $2.78^{* *}$ & .82 \\
\hline $\begin{array}{l}\text { MT viso- } \\
\text { espacial }\end{array}$ & Amplitud & $\begin{array}{l}\text { Control } \\
\text { Clínico }\end{array}$ & $\begin{array}{l}30 \\
19\end{array}$ & $\begin{array}{l}3.83 \\
2.42\end{array}$ & $\begin{array}{l}1.02 \\
.61\end{array}$ & $\begin{array}{l}.19 \\
.14\end{array}$ & $5.42^{* * *}$ & 1.59 \\
\hline $\begin{array}{l}\text { Inhibición com- } \\
\text { portamental }\end{array}$ & Tiempo de frenado (TF) & $\begin{array}{l}\text { Control } \\
\text { Clínico }\end{array}$ & $\begin{array}{l}30 \\
19\end{array}$ & $\begin{array}{l}410.74 \\
737.46\end{array}$ & $\begin{array}{l}147.25 \\
117.57\end{array}$ & $\begin{array}{l}26.88 \\
26.97\end{array}$ & $8.15^{\star \star *}$ & 2.39 \\
\hline $\begin{array}{l}\text { Inhibición } \\
\text { cognitiva }\end{array}$ & $\begin{array}{l}\text { TR medio - diferencia entre PI y } \\
\text { PN } \\
\text { Precisión - diferencia entre PI y } \\
\text { PN }\end{array}$ & $\begin{array}{l}\text { Control } \\
\text { Clínico } \\
\text { Control } \\
\text { Clínico }\end{array}$ & $\begin{array}{l}30 \\
19 \\
30 \\
19\end{array}$ & $\begin{array}{c}33.22 \\
-12.27 \\
13.73 \\
2.32\end{array}$ & $\begin{array}{l}378.60 \\
478.86 \\
\\
34.85 \\
30.77\end{array}$ & $\begin{array}{c}69.12 \\
109.86 \\
\\
6.36 \\
7.06\end{array}$ & $\begin{array}{l}0.37 \\
1.16\end{array}$ & - \\
\hline $\begin{array}{l}\text { Inhibición } \\
\text { perceptual }\end{array}$ & $\begin{array}{l}\text { Precisión } \\
32 \text { distractores } \\
\text { TR medio } \\
32 \text { distractores } \\
\text { Dif. en Precisión entre condi- } \\
\text { ción } 4 \text { y } 32 \text { distractores } \\
\text { Dif .en TR entre condición } 4 \text { y } \\
32 \text { distractores }\end{array}$ & $\begin{array}{l}\text { Control } \\
\text { Clínico } \\
\text { Control } \\
\text { Clínico } \\
\text { Control } \\
\text { Clínico } \\
\text { Control } \\
\text { Clínico }\end{array}$ & $\begin{array}{l}30 \\
18 \\
30 \\
18 \\
30 \\
18 \\
30 \\
18\end{array}$ & $\begin{array}{c}84.33 \\
84.81 \\
\\
1571.13 \\
1863.78 \\
\\
9.83 \\
9.08 \\
-460.53 \\
-594.94\end{array}$ & $\begin{array}{c}11.04 \\
9.374 \\
\\
344.48 \\
412.82 \\
\\
10.30 \\
8.77 \\
\\
265.35 \\
371.12\end{array}$ & $\begin{array}{l}2.02 \\
2.21 \\
62.89 \\
97.30 \\
\\
1.88 \\
2.07 \\
48.45 \\
87.47\end{array}$ & $\begin{array}{l}0.15 \\
2.64^{*} \\
0.26 \\
1.46\end{array}$ & - \\
\hline $\begin{array}{l}\text { Flexibilidad } \\
\text { cognitiva }\end{array}$ & $\begin{array}{l}\text { TR medio en BM } \\
\text { Precisión en BM } \\
\text { Coste de cambio local en TR } \\
\begin{array}{l}\text { Coste de cambio local en Preci- } \\
\text { sión }\end{array}\end{array}$ & $\begin{array}{l}\text { Control } \\
\text { Clínico } \\
\text { Control } \\
\text { Clínico } \\
\text { Control } \\
\text { Clínico } \\
\text { Control } \\
\text { Clínico }\end{array}$ & $\begin{array}{l}30 \\
19 \\
30 \\
19 \\
30 \\
19 \\
30 \\
19\end{array}$ & $\begin{array}{c}579.92 \\
874.48 \\
\\
95.07 \\
73.89 \\
\\
75.92 \\
-373.86 \\
\\
-.17 \\
-.64\end{array}$ & $\begin{array}{c}82.53 \\
112.42 \\
\\
13.30 \\
19.29 \\
\\
70.38 \\
364.82 \\
.13 \\
6.38\end{array}$ & $\begin{array}{c}15.07 \\
25.80 \\
\\
2.43 \\
4.43 \\
\\
12.85 \\
83.70 \\
.02 \\
1.46\end{array}$ & $\begin{array}{c}10.56^{* * *} \\
4.19^{* * *} \\
6.60^{* * *} \\
.40\end{array}$ & $\begin{array}{l}3.09 \\
1.33 \\
1.28\end{array}$ \\
\hline
\end{tabular}

${ }^{*} p<.05$

${ }^{* *} p<.01$

${ }^{* * *} p<.001$ 
TABLA 2

ANÁLISIS DISCRIMINANTE PARA LA FUNCIÓN 1, MEDIANTE EL MÉTODO DE INCLUSIÓN DE VARIABLES TODAS JUNTAS, PARA LA IDENTIFICACIÓN DE MEDIDAS EJECUTIVAS ASOCIADAS A LA PERTENENCIA A LOS GRUPOS CLÍNICO Y CONTROL

\begin{tabular}{|c|c|c|}
\hline & $\begin{array}{l}\text { Coeficientes } \\
\text { estandarizados }\end{array}$ & $\begin{array}{c}\text { Coeficientes de } \\
\text { estructura }\end{array}$ \\
\hline $\begin{array}{l}\text { MT verbal. Amplitud } \\
\text { MT visoespacial. Amplitud } \\
\text { Inhibición comportamental. TF } \\
\text { Inhibición perceptual. TR medio } 32 \text { distractores } \\
\text { FC. TR medio en BM } \\
\text { FC. Precisión en BM } \\
\text { FC. Coste de cambio local en TR }\end{array}$ & $\begin{array}{r}.088 \\
.504 \\
-.270 \\
.090 \\
-.697 \\
.382 \\
.325\end{array}$ & $\begin{array}{l}-.168 \\
.332 \\
-.500 \\
-.169 \\
-.662 \\
.272 \\
.408\end{array}$ \\
\hline $\begin{array}{l}\text { Autovalor } \\
\lambda \\
\text { Correlación canónica }\end{array}$ & $\chi^{2}=$ & .001 \\
\hline
\end{tabular}

\section{TABLA 3}

RESULTAdOS DE LA CLASIFICACIÓN DEL ANÁLISIS DISCRIMINANTE EN FUNCIÓN DE LA FRECUENCIA Y PORCENTAJE DE CORRECTOS CLASIFICADOS

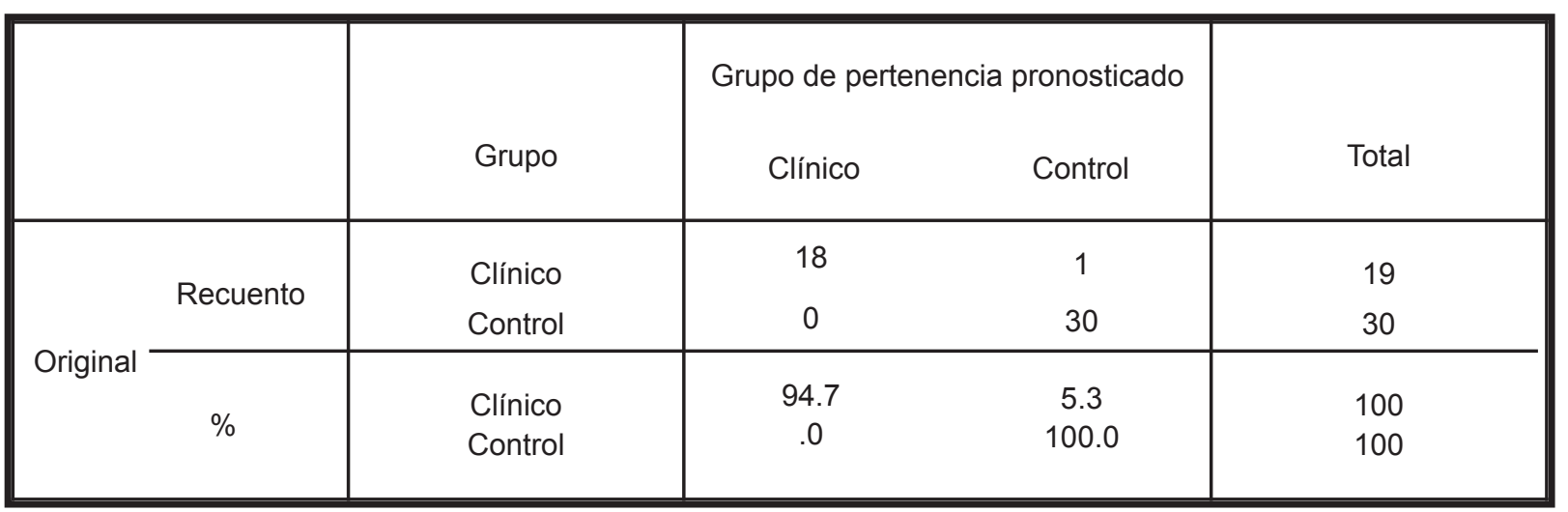

Nota: Clasificados correctamente el $98 \%$ de los casos agrupados originales 
TABLA 4

CURVA ROC: ÁREA POR DEBAJO DE LA CURVA PARA LAS VARIABLES CON MAYOR PODER DISCRIMINANTE

\begin{tabular}{|c|c|c|c|c|c|c|}
\hline \multirow{2}{*}{ Proceso } & \multirow{2}{*}{ Índice } & \multirow{2}{*}{ ABC } & \multirow{2}{*}{$\begin{array}{c}\text { Error } \\
\text { Estándar }\end{array}$} & \multirow{2}{*}{ Sig. } & \multicolumn{2}{|c|}{$\begin{array}{c}\text { Intervalo de } \\
\text { confianza del } 95 \%\end{array}$} \\
\hline & & & & & $\begin{array}{l}\text { Límite } \\
\text { inferior }\end{array}$ & $\begin{array}{l}\text { Límite } \\
\text { superior }\end{array}$ \\
\hline MT verbal & Amplitud & .708 & .074 & .017 & .563 & .854 \\
\hline MT visoespacial & Amplitud & .856 & .055 & .000 & .748 & .963 \\
\hline & TR medio & & & & & \\
\hline Inhibición perceptual & Condición 32 distractores & .706 & .075 & .018 & .560 & .853 \\
\hline Inhibición comportamental & Tiempo de frenado & .978 & .017 & .000 & .945 & 1.000 \\
\hline \multirow{3}{*}{$\begin{array}{l}\text { Flexibilidad } \\
\text { Cognitiva }\end{array}$} & Precisión en BM & .907 & .048 & .000 & .810 & .988 \\
\hline & TR medio en BM & .989 & .010 & .000 & .968 & 1.000 \\
\hline & Coste de cambio local en TR & .895 & .074 & .000 & .757 & 1.000 \\
\hline
\end{tabular}

Nota:

$A B C$ : Área bajo la curva

Valores del ABC ROC comprendidos entre .5 y .7 indican baja exactitud, entre .7 y .9 moderada, según el propósito y valores mayores de .9 indican exactitud alta 
Richard's, Vernucci, Zamora, Canet Juric, Introzzi y Guardia

TABLA 5

ÁREA BAJO LA CURVA, PUNTO DE CORTE, SENSIBILIDAD Y ESPECIFICIDAD PARA CADA ÍNDICE DE DESEMPEÑO

\begin{tabular}{l|l|c|c|c|c|}
\hline \multicolumn{1}{|c|}{ Proceso } & \multicolumn{1}{|c|}{ Índice } & ABC & $\begin{array}{c}\text { Punto de } \\
\text { corte }\end{array}$ & $\begin{array}{c}\text { Sensibilidad } \\
\%\end{array}$ & $\begin{array}{c}\text { Especificidad } \\
\%\end{array}$ \\
\hline MT verbal & Amplitud & .708 & $4^{\mathrm{a}}$ & 84.2 & 46.7 \\
MT visoespacial & Amplitud & .856 & $3^{\mathrm{a}}$ & 94.7 & 70 \\
\hline Inhibición perceptual & $\begin{array}{l}\text { TR medio. Condición 32 } \\
\text { distractores }\end{array}$ & .706 & $1615^{\mathrm{b}}$ & 72.2 & 63.3 \\
\hline $\begin{array}{l}\text { Inhibición comportamen- } \\
\text { tal }\end{array}$ & Tiempo de frenado & .978 & $537^{\mathrm{b}}$ & 94.4 & 93.3 \\
\hline Flexibilidad \\
Cognitiva
\end{tabular}

\section{Notación}

ABC Área bajo la curva

a ítems

${ }^{b}$ milisegundos

${ }^{\mathrm{c}}$ porcentaje 
Validez de grupos contrastados en procesos ejecutivos

FIGURA 1

CURVA ROC. ÍNDICES DE SENSIBILIDAD Y ESPECIFICIDAD DE LOS VALORES DE AMPLITUD DE MEMORIA DE TRABAJO VERBAL Y VISOESPACIAL

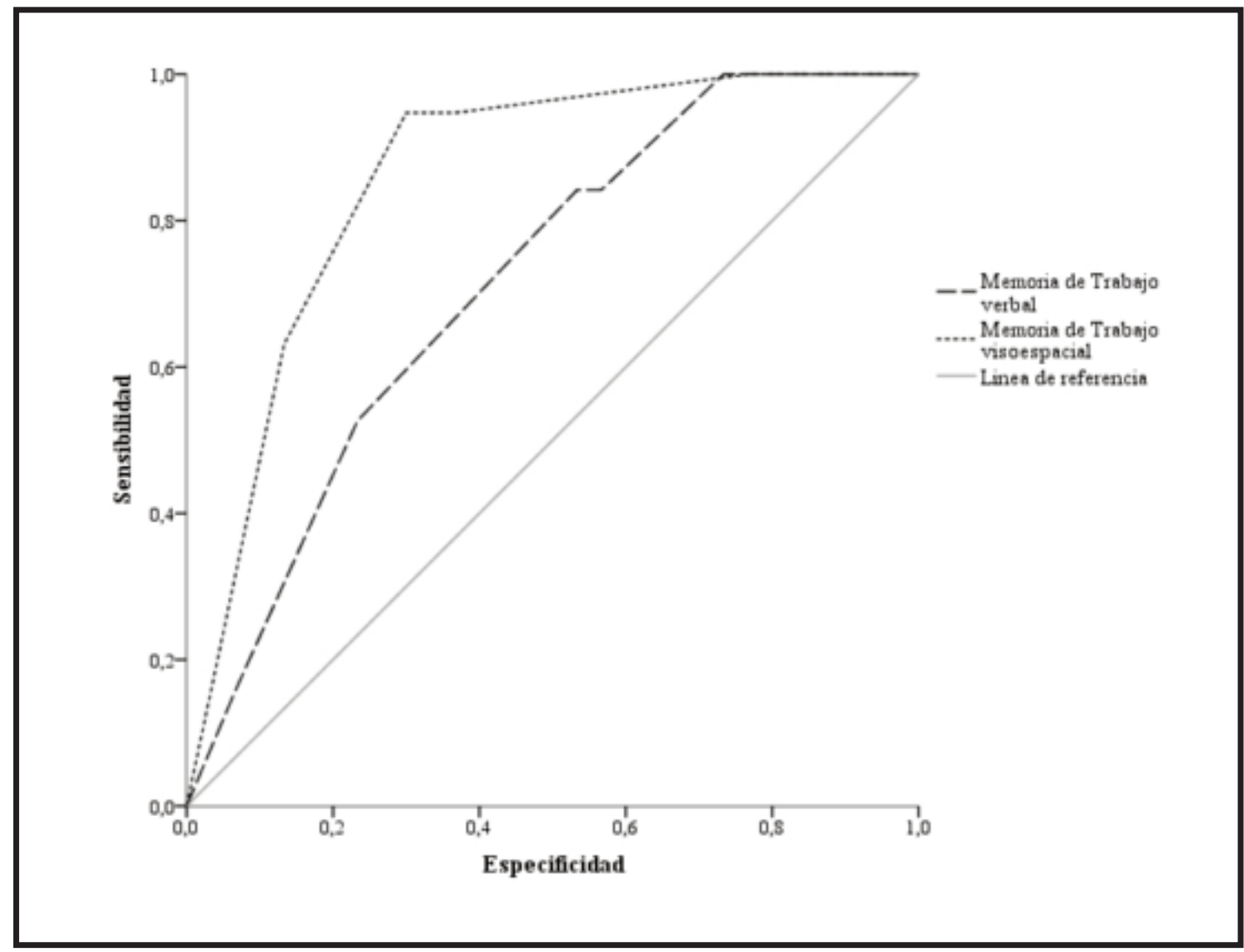


FIGURA 2

CURVA ROC. ÍNDICES DE SENSIBILIDAD Y ESPECIFICIDAD DE LOS PROCESOS INHIBITORIOS: TR MEDIO DE LA TAREA DE INHIBICIÓN PERCEPTUAL CON 32 DISTRACTORES Y TIEMPO DE FRENADO DE LA TAREA DE INHIBICIÓN COMPORTAMENTAL

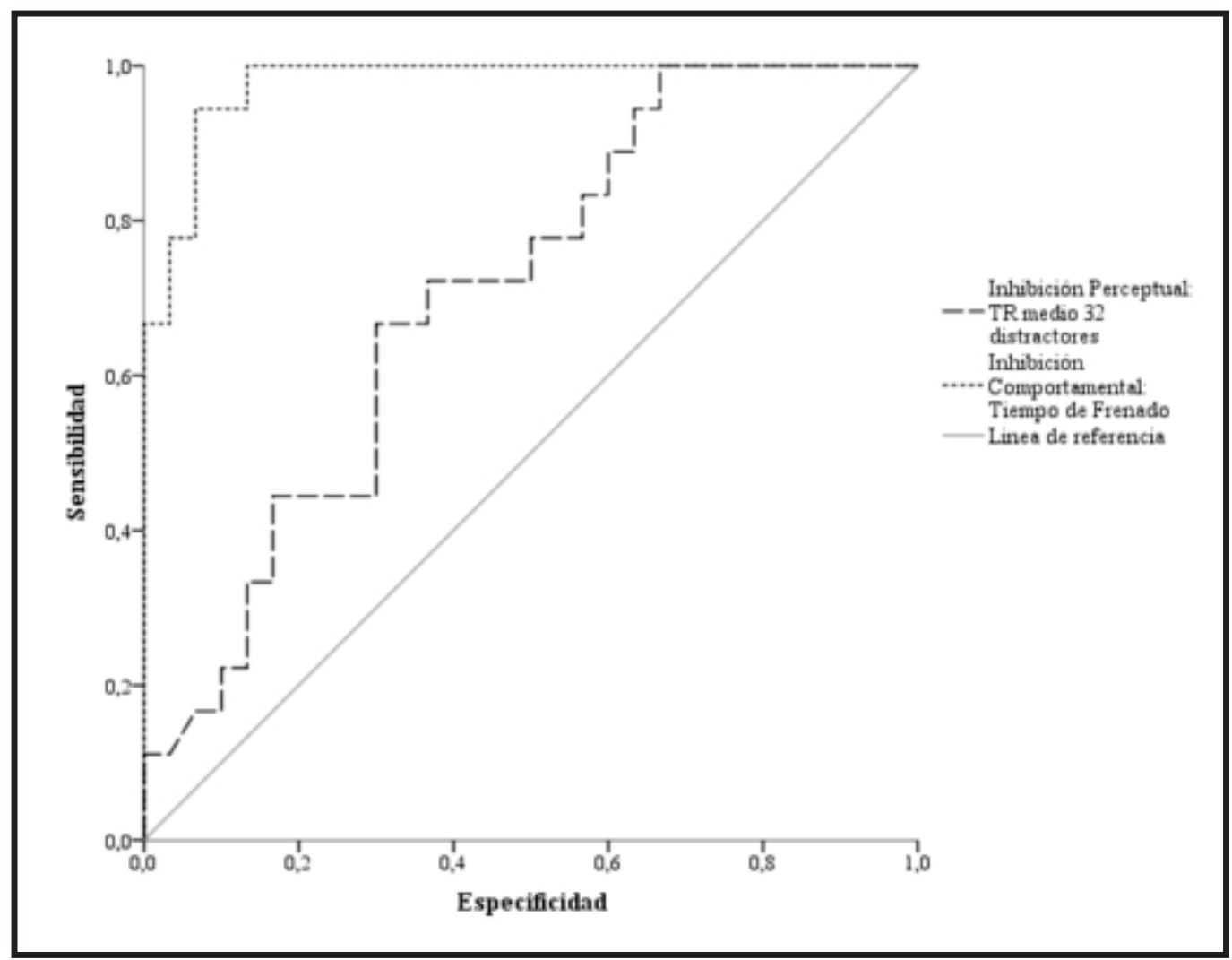


Validez de grupos contrastados en procesos ejecutivos

FIGURA 3

CuRva ROC. Índices de Sensibilidad y EsPeCIFICIDAd DE FleXIBILIDAd Cognitiva: TR MEdio en el BLOQUE MIXTO, PRECISIÓN EN EL BLOQUE MIXTO Y EFECTO DE COSTE DE CAMBIO LOCAL EN TR

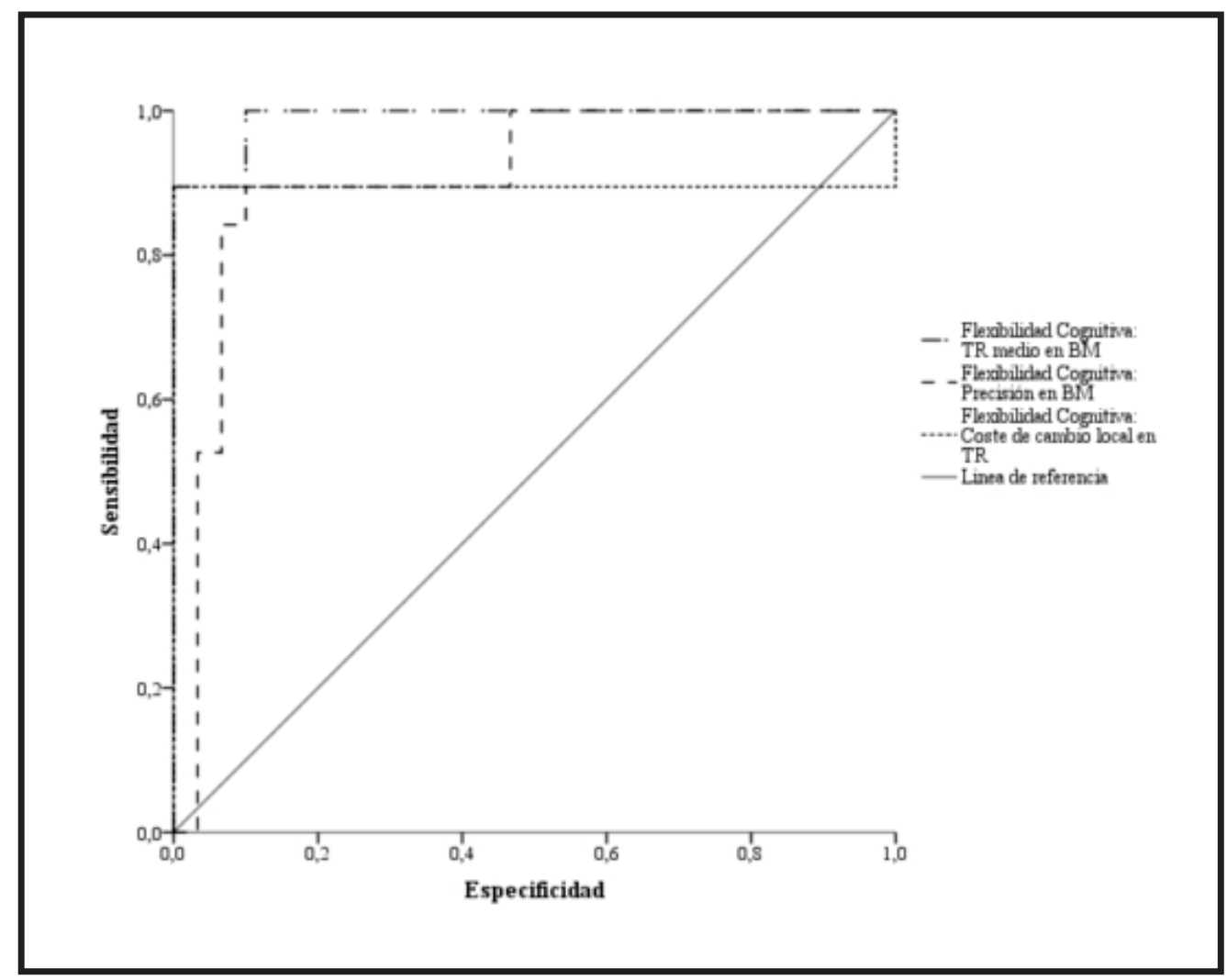




\section{REFERENCIAS BIBLIOGRÁFICAS}

American Psychiatric Association (2013). Diagnostic and statistical manual of mental disorders (DSM-5). Arlington, VA, Estados Unidos: American Psychiatric Publishing.

Arán Filippetti, V. \& Mías, C. (2009). Neuropsicología del trastorno por déficit de atención / hiperactividad: Subtipos predominio déficit de atención y predominio hiperactivo-impulsivo [Neuropsychology of attention deficit disorder / hyperactivity disorder: Prevalence attention deficit and hyperactive-impulsive predominance subtypes]. Revista Argentina de Neuropsicología, 13, 14-28.

Barkley, R.A. (1997). Behavioral inhibition, sustained attention, and executive functions: Constructing a unifying theory of ADHD. Psychological Bulletin, 121(1), 65-94. http://dx.doi. org/10.10.1037/0033-2909.121.1.65

Barkley, R.A., Edwards, G., Laneri, M., Fletcher, K. \& Metevia, L. (2001). Executive functioning, temporal discounting, and sense of time in adolescents with attention deficit hyperactivity disorder (ADHD) and oppositional defiant disorder (ODD). Journal of Abnormal Child Psychology, 29(6), 541-556.

Bédard, A.C.V., Trampush, J.W., Newcorn, J.H. \& Halperin, J.M. (2010). Perceptual and motor inhibition in adolescents / young adults with childhood-diagnosed ADHD. Neuropsychology, 24(4), 424-434. http://dx.doi.org/10.10.1 037/a0018752

Brocki, K.C. \& Bohlin, G. (2006). Developmental change in the relation between executive functions and symptoms of ADHD and co-occurring behaviour problems. Infant and Child Development, 15, 19-40.

Bull, R. \& Lee, K. (2014). Executive functioning and mathematics achievement. Child Development Perspectives, 8(1), 36-41. http://dx.doi.or g/1 0.10.1111/cdep.12059

Cain, K.E., Oakhill, J. \& Bryant, P.E. (2004). Children's reading comprehension ability: Concurrent prediction by working memory, verbal ability, and component skills. Journal of Edu- cational Psychology, 96(1), 31-42. http://dx.d oi.org/10.10.1037/0022-0663.96.1.31

Canet Juric, L., Introzzi, I., \& Burin, D.I. (2015). Desarrollo de la capacidad de memoria de trabajo: Efectos de interferencia inter e intra dominio en niños de edad escolar [Development of working memory capacity: School children interference inter-domain and intra-domain effects]. Revista Argentina de Ciencias del Comportamiento, 7(1), 26-37.

Conway, A.R., Cowan, N., Bunting, M.F., Therriault, D.J. \& Minkoff, S.R. (2002). A latent variable analysis of working memory capacity, short-term memory capacity, processing speed, and general fluid intelligence. Intelligence, 30 (2), 163-183. http://dx.doi.org/10.10.1016/s01 60-2896(01)00096-4

Davidson, M.C., Amso, D., Anderson, L.C. \& Diamond, A. (2006). Development of cognitive control and executive functions from 4 to 13 years: Evidence from manipulations of memory, inhibition, and task switching. Neuropsychologia, 44(11), 2037-2078. http://dx.doi.org/ 10.0.1016/j.neuropsychologia.2006.02.006

Diamond, A. (2013). Executive functions. Annual Review of Psychology, 64, 135. http://dx.doi. org/10.10.1146/annurev-psych-113011-143750

Doyle, A.E. (2006). Executive functions in attention-deficit / hyperactivity disorder. The Journal of Clinical Psychiatry, 67(8), 21-26.

Engelhardt, P.E., Nigg, J.T., Carr, L.A. \& Ferreira, F. (2008). Cognitive inhibition and working memory in attention-deficit / hyperactivity disorder. Journal of Abnormal Psychology, 117(3), 591-605. http://dx.doi.org/10.10.1037/a00125 93

Friedman, N.P. \& Miyake, A. (2004). The relations among inhibition and interference control functions: A latent-variable analysis. Journal of Experimental Psychology: General, 133(1), 101135. http://dx.doi.org/10.10.1037/0096-34 45.1 33.1.101

Fuggetta, G.P. (2006). Impairment of executive functions in boys with attention deficit / hyperactivity disorder. Child Neuropsychology, 12(1), 1-21. http://dx.doi.org/10.10.1080/092970405 00203418 
Gandolfi, E., Viterbori, P., Traverso, L. \& Usai, M. C. (2014). Inhibitory processes in toddlers: A latent-variable approach. Frontiers in Psychology, 5, 381. http://dx.doi.org/10.3389/fpsyg.2 014.00381

Hajian-Tilaki, K. (2013). Receiver operating characteristic (ROC) curve analysis for medical diagnostic test evaluation. Caspian Journal of Internal Medicine, 4(2), 627.

Hale, S., Bronik, M.D. \& Fry, A.F. (1997). Verbal and spatial working memory in school-age children: Developmental differences in susceptibility to interference. Developmental Psychology, 33(2), 364. http://dx.doi.org/10.1037/0 012-1649.33.2.364

Hofmann, W., Schmeichel, B.J. \& Baddeley, A.D. (2012). Executive functions and self-regulation. Trends in Cognitive Sciences, 16(3), 174-180. http://dx.doi.org/10.1016/j.tics.2012.01.006

Howard, S.J., Johnson, J. \& Pascual-Leone, J. (2014). Clarifying inhibitory control: Diversity and development of attentional inhibition. Cognitive Development, 31, 1-21. http://dx.doi. org/10.1016/j.cogdev.2014.03.001

Introzzi, I., Canet-Juric, L., Montes, S., López, S. \& Mascarello, G. (2015). Inhibitory processes and cognitive flexibility: Evidence for the theory of attentional inertia. International Journal of Psychological Research, 8(2), 61-75.

Logan, G.D. (1994). On the ability to inhibit thought and action: A user's guide to the stop signal paradigm. En D. Dagenbach \& T.H. Carr (Eds.), Inhibitory processes in attention, memory, and language (pp. 189-239). San Diego, C A: Academic Press.

McAuley, T., Crosbie, J., Charach, A. \& Schachar, R. (2014). The persistence of cognitive deficits in remitted and unremitted ADHD: A case for the state-independence of response inhibition. Journal of Child Psychology and Psychiatry, 55(3), 292-300. http://dx.doi.org/10.1111/jcpp. 12160

Mischel, W., Ayduk, O., Berman, M.G., Casey, B.J., Gotlib, I.H., Jonides, J. et al. (2011). 'Willpower' over the life span: Decomposing self- regulation. Social Cognitive and Affective Neuroscience, 6(2), 252-256. http://dx.doi.org/10. 1093/scan/nsq081

Miyake, A. \& Friedman, N.P. (2012). The nature and organization of individual differences in executive functions four general conclusions. Current Directions in Psychological Science, 21(1), 8-14. http://dx.doi.org/10.1177/0963721 411429458

Montero, I. \& León, O.G. (2007). Guía para nombrar los estudios de investigación en Psicología [Guide for naming research studies in Psychology]. International Journal of Clinical and Health Psychology, 7(3), 847-862.

Navarro-Guzmán, J.I. (2005). Índice de status social [Social status index]. Cádiz, España: Universidad de Cádiz.

Nigg, J.T. (2001). Is ADHD a disinhibitory disorder? Psychological Bulletin, 127(5), 571. http://dx.doi.org/10.1037/0033-2909.127.5.571

Oberauer, K. (2001). Removing irrelevant information from working memory: A cognitive aging study with the modified Sternberg task. Journal of Experimental Psychology: Learning, Memory, and Cognition, 27(4), 948. http://dx. doi.org/10.1037/0278-7393.27.4.948

Richard's, M., Introzzi, I., Zamora, E. \& Vernucci, S. (2016). Analysis of internal and external validity criteria for a computerized visual search task. A pilot study. Applied Neuropsychology: Child, 1-10. http://dx.doi.org/10.1080/2162296 5.2015.1083433.

Roberts, B.A., Martel, M.M. \& Nigg, J.T. (2013). Are there executive dysfunction subtypes within ADHD? Journal of Attention Disorders. http://dx.doi.org/10.1177/1087054713510349. First published on November 8, 2013

Romero-Ayuso, D.M., Maestú, F., González-Marqués, J., Romo-Barrientos, C. \& Andrade, J.M. (2006). Disfunción ejecutiva en el trastorno por déficit de atención con hiperactividad en la inDisfunción ejecutiva en el trastorno por déficit de atención con hiperactividad en la infancia. fancia [Executive dysfunction in attention deficit hyperactivity disorder in childhood]. Revista de Neurología, 42(5), 265-271. 
Rubia, K., Smith, A.B., Taylor, E. \& Brammer, M. (2007). Linear age-correlated functional development of right inferior fronto-striato-cerebellar networks during response inhibition and anterior cingulate during error-related processes. Human Brain Mapping, 28(11), 1163-1177. http://dx.doi.org/10.1002/h bm. 20347

Rubiales, J. (2014). Perfil ejecutivo en niños con Trastorno por Déficit de Atención con Hiperactividad [Executive functioning in children with Attention Deficit Hyperactivity Disorder]. Revista Iberoamericana de Diagnóstico y Evaluación Psicológica - RIDEP, 38(2), 31-54.

Scahill, L. \& Schwab-Stone, M. (2000). Epidemiology of ADHD in school-age children. Child and Adolescent Psychiatric Clinics of North America, 9(7), 541-555.

Senderecka, M., Grabowska, A., Gerc, K., Szewczyk, J. \& Chmylak, R. (2012). Event-related potentials in children with attention deficit hyperactivity disorder: An investigation using an auditory oddball task. International Journal of Psychophysiology, 85(1), 106-115. http://dx doi.org/10.1016/j.ijpsycho.2011.5.006

Swets, J.A. (1988). Measuring the accuracy of diagnostic systems. Science, 240(4857), 1285-1293.

Treisman, A. \& Gelade, G. (1980). A featureintegration theory of attention. Cognitive Psychology, 12(1), 97-136. http://dx.doi.org/10.1 016/0010-0285(80)90005-5

Vadaga, K.K., Blair, M., \& Li, K.Z.H. (2016). Are age-related differences uniform across different inhibitory functions? Journals of Gerontology, Series B: Psychological Sciences and Social Sciences, 71(4), 641-649. http://dx.doi.or g/10.1 093/geronb/gbv002

Willcutt, E.G., Doyle, A.E., Nigg, J.T., Faraone, S.V. \& Pennington, B.F. (2005). Validity of the executive function theory of attention-deficit/ hyperactivity disorder: A meta-analytic review. Biological Psychiatry, 57(11), 1336-1346. http: //dx.doi.org/10.1016/j.biopsych.2005.02.006

Zhou, X.H., Obuchowski, N.A. \& McClish, D.K. (2002). Statistical methods in diagnostic medicine. New York: Wiley Interscience.

Centro Interdisciplinario de Investigaciones en Psicología Matemática y Experimental Dr. Horacio J.A. Rimoldi (CIIPME)

Ciudad Autónoma de Buenos Aires

Instituto de Psicología Básica, Aplicada y Tecnología (IPSIBAT) Centro de Investigación en Procesos Básicos, Metodología y Educación (CIMEPB) Facultad de Psicología

Universidad Nacional de Mar del Plata (UNMdP) Consejo Nacional de Investigaciones Cientificas y Técnicas (CONICET) República Argentina

Departamento de Metodología de las Ciencias del Comportamiento Facultad de Psicología Universidad de Barcelona (UB) e Instituto de Investigación de Cerebro, Cognición y Conducta (IR3C) España

Fecha de recepción: 10 de marzo de 2016 Fecha de aceptación: 26 de septiembre de 2016 